\title{
Controversies of renin-angiotensin system inhibition during the COVID-19 pandemic
}

'Section of Nephrology, Department of Pediatrics, Wake Forest School of Medicine and Brenner Children's Hospital, Winston Salem, NC, USA.

${ }^{2}$ Department of Epidemiology and Prevention, Division of Public Health Sciences, Wake Forest School of Medicine, Winston Salem, NC, USA.

${ }^{3}$ Department of SurgeryHypertension and Vascular Research, Wake Forest School of Medicine, Winston Salem, NC, USA.

${ }^{4}$ Cardiovascular Sciences Center, Wake Forest School of Medicine, Winston Salem NC, USA.

${ }^{5}$ Department of NonCommunicable Disease Epidemiology, London School of Hygiene and Tropical Medicine, London, UK.

${ }^{6}$ Division of Nephrology, Department of Medicine, Duke University School of Medicine and Renal Section, Durham VA Health Care System, Durham, NC, USA.

${ }^{7}$ Duke Clinical Research Institute, Durham, NC, USA.

${ }^{8}$ Division of Nephrology, Department of Medicine, University of Ottawa, Ottawa Ontario, Canada.

凶e-mail: matthew.sparks@ duke.edu

https://doi.org/10.1038/ s41581-020-0279-4

Andrew M. South ${ }^{1,2,3,4}$, Laurie Tomlinson ${ }^{5}$, Daniel Edmonston ${ }^{6,7,7}$, Swapnil Hiremath ${ }^{8}$ and Matthew A. Sparks ${ }^{6 凶}$

The current COVID-19 pandemic is associated with unprecedented morbidity and mortality. Early reports suggested an association between disease severity and hypertension but did not account for sources of confounding. However, the responsible virus - SARS-CoV-2 - gains entry to host cells via angiotensin-converting enzyme 2 (ACE2), highlighting the need to understand the relationship between the virus and the renin-angiotensin system (RAS) and how this might be affected by RAS inhibitors.

Coronavirus disease 2019 (COVID-19) is caused by severe acute respiratory syndrome coronavirus 2 (SARS-CoV-2). This virus has a higher transmission rate than SARS-CoV, which emerged in 2002, and, in some patients, COVID-19 results in acute respiratory distress syndrome with high morbidity and mortality. Early reports suggested that patients with severe COVID-19 were more likely to have a history of hypertension, chronic kidney disease (CKD), cardiovascular disease (CVD) or diabetes mellitus (DM) than those with milder disease ${ }^{1}$. However, these preliminary findings did not account for potential sources of bias and confounding, including age, sex, baseline pulmonary disease, co-morbid conditions or baseline medication use. Thus, the risk attributable to hypertension, CKD, CVD and $\mathrm{DM}$ is yet to be confirmed. Consideration of age as a potential confounder is particularly important, as the prevalence of hypertension increases dramatically with age. In the absence of age-adjusted or stratified analyses of the incidence or severity of COVID-19, it is important to consider potential plausible mechanisms by which hypertension or hypertension medications might influence COVID-19 severity. The mechanisms underlying the purported association between COVID-19 severity and hypertension are not clear, but some evidence points towards a pathogenic role for the renin-angiotensin system (RAS), as it is tied directly to both viral transmission and hypertension.

\section{The renin-angiotensin system}

Angiotensin-converting enzyme 2(ACE2) is an enzyme within the RAS that is expressed on the cell surface of type 2 alveolar epithelial cells in the lungs, as well as on cells in many other tissues. It also acts as the receptor for the SARS-CoV-2 spike protein, through which the virus gains entry to host cells. ACE2 is also the receptor for the previously described SARS-CoV; however, the affinity of SARS-CoV-2 for ACE2 is 10-20-fold higher than that of SARS-CoV, which could explain its higher transmissibility ${ }^{2}$. Binding of the spike protein to ACE2, along with proteolytic cleavage of ACE2 by transmembrane serine protease 2 (TMPRSS2), facilitates entry of the virus into cells, viral replication and cell-to-cell transmission ${ }^{2}$.

ACE2 is a crucial counter-regulatory component of the RAS and shares approximately $60 \%$ homology with ACE. ACE2 converts angiotensin II (Ang II) into Ang-(1-7), which acts on the Mas receptor, expressed on a variety of cell lineages in many tissues relevant to cardiovascular disease (including type 2 alveolar epithelial cells), to modestly lower blood pressure through vasodilation and by promoting kidney sodium and water excretion but also to attenuate inflammation through the production of nitric oxide ${ }^{3}$. These effects directly oppose those induced by ACE-Ang II signalling, whereby ACE converts Ang I into Ang II, which acts at the type 1 angiotensin receptor (AT1R) to increase blood pressure by inducing vasoconstriction, increasing kidney reabsorption of sodium and water, and increasing oxidative stress to promote inflammation and fibrosis ${ }^{4}$. Components of both RAS pathways are co-expressed in the majority of tissues and organ systems in humans and act in both a paracrine and an autocrine manner; thus, the balance between these pathways determines - at least in part - whether or not tissue injury will occur in response to a stimulus, especially in the heart and kidneys. Extrapolating data from SARS-CoV to SARS-CoV-2 and COVID-19 suggests that increased activity of ACEAng II relative to that of ACE2-Ang-(1-7) might drive acute lung injury in SARS-CoV-2 and COVID-19 (REF.5). 
Binding of the SARS-CoV-2 spike protein to ACE2, followed by proteolytic cleavage and viral entry, is thought to suppress expression of ACE2. We hypothesize that this suppression of ACE2 occurs as a consequence of increased internalization and shedding of ACE2 from the cell surface, which leads to decreased tissue ACE2 and decreased Ang-(1-7) generation, and consequently increased Ang II levels. Ang-(1-7) levels are further reduced as ACE converts Ang-(1-7) into less biologically active peptides (FIG. 1). As shown in an experimental model of SARS-CoV, this process can drive an Ang IIAT1R-mediated inflammatory response in the lungs and potentially induce direct parenchymal injury ${ }^{5}$.

Concern has emerged that ACE inhibitors and angiotensin-receptor blockers (ARBs), which are prescribed to millions of patients worldwide, could theoretically increase the risk of SARS-CoV-2 infection and more severe COVID-19 owing to the role of ACE2 as the viral binding site. Although ACE inhibitors and ARBs do not directly affect ACE2 activity, studies in experimental
No RAS inhibitor

Hypothesis 1 - RAS inhibition is harmful in COVID-19

Viral binding

SARS-CoV-2

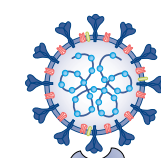

ACE2

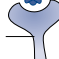

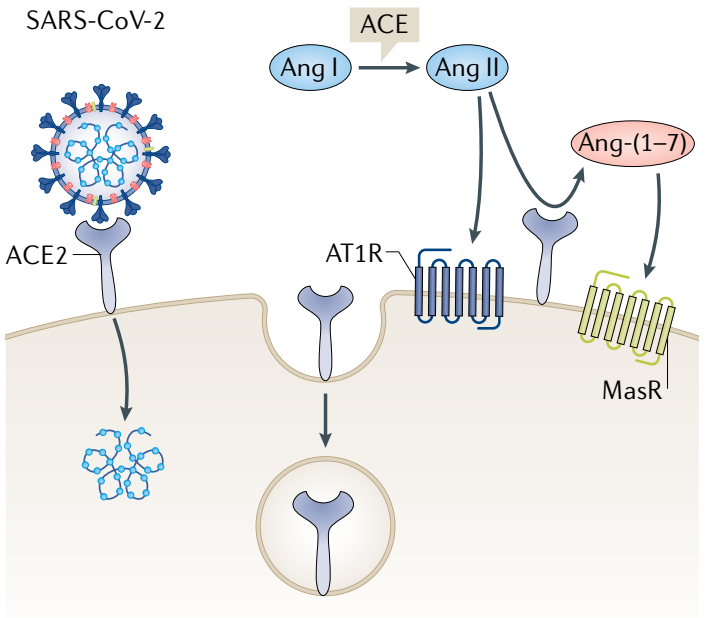

Hypothesis 2 - RAS inhibition is protective in COVID-19

Increased lung injury

Hethesis $2-$ RAS inhibition is

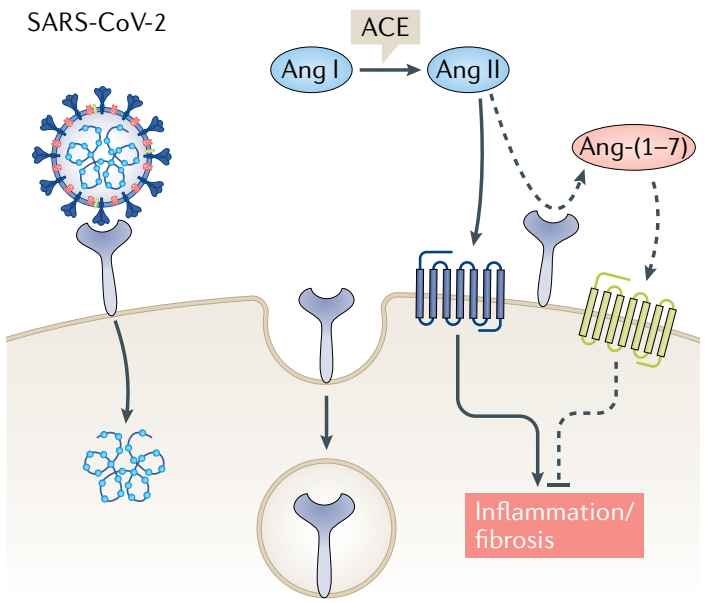

RAS inhibitor

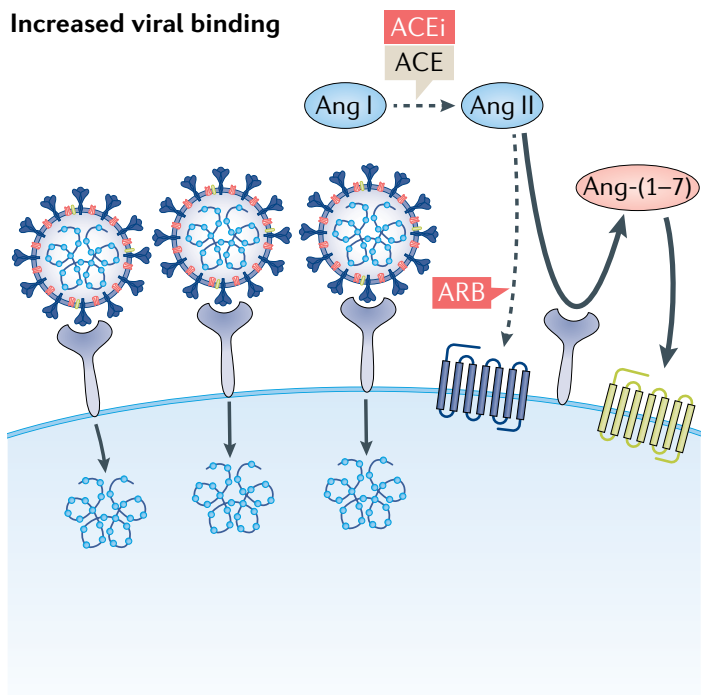

Decreased lung injury

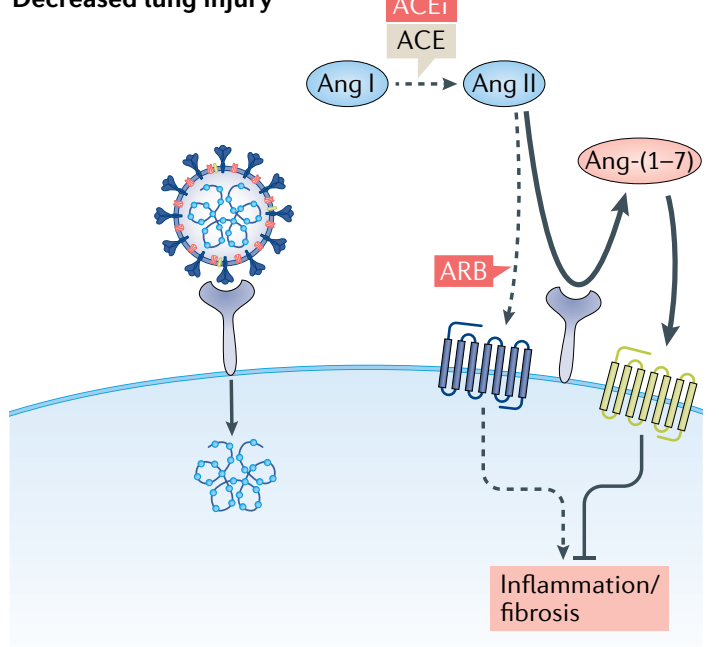

Fig. 1 | Possible effects of renin-angiotensin system inhibition on COVID-19. The competing hypothetical mechanisms by which inhibition of the renin-angiotensin system (RAS) with an angiotensin-converting enzyme inhibitor (ACEi) or angiotensin-receptor blocker (ARB) might be harmful (upper panels) or protective (lower panels) in COVID-19. Hypothesis 1: severe acute respiratory syndrome coronavirus 2 (SARS-CoV-2) gains entry into the cell by binding to angiotensin-converting enzyme 2 (ACE2; upper left panel). The addition of an ACEi or ARB could increase ACE2 abundance and thus enhance viral entry (upper right panel). Hypothesis 2: angiotensin II (Ang II) drives lung injury by activating the type 1 angiotensin receptor (AT1R), causing inflammation and fibrosis (lower left panel). Diminishing production of Ang II with an ACEi or blocking Ang II-AT1R actions with an ARB enhances the generation of Ang-(1-7) by ACE2 and activation of the Mas receptor (MasR), which attenuates inflammation and fibrosis and therefore attenuates lung injury. 
animal models showing that these agents can upregulate the expression and activity of ACE2 in heart and kidney tissue $e^{6,7}$ have prompted concerns about the potential increased susceptibility of patients receiving these drugs to SARS-CoV-2 infection and/or COVID-19 severity (FIG. 1). However, studies that show an effect of ACE inhibitors or ARBs on ACE2 expression or activity in lung are lacking, and, importantly, other studies in animals and humans have been unable to demonstrate that upregulation of ACE2 occurs in response to ACE inhibitors or $\mathrm{ARBs}^{8,9}$. Furthermore, experimental models of acute lung injury, including a model of SARS-CoV infection, suggest that ARBs may mitigate COVID-19 by attenuating Ang II-mediated acute lung injury by blocking AT1R ${ }^{5}$. Finally, to our knowledge, no available human studies support the hypothesis that baseline use of ACE inhibitors or ARBs increases the risk of SARS-CoV-2 infection or the development of severe outcomes in patients with COVID-19.

\section{Clinical implications}

ACE inhibitors and ARBs are prescribed for several indications including hypertension, proteinuric CKD and heart failure. The consequences of stopping these drugs will vary depending on the condition. Although other drug classes may be available for the management of hypertension, proteinuric CKD and heart failure with impaired left ventricular function are compelling indications for these drugs. Moreover, individuals taking these drugs may not know the indication for which the drugs were prescribed and/or their clinical condition may have changed since the drugs were initiated. For example, patients taking ACE inhibitors or ARBs for hypertension may have since developed impaired left ventricular function and stopping these drugs could lead to clinical symptoms of heart failure. Concern about inappropriate cessation of drugs prompted the United Kingdom to release national consensus guidelines in 2019 for patients with heart failure, which encourage patients to continue these drugs unless stopped by clinicians when deemed necessary (for example, following development of hypotension or hyperkalaemia). Similar issues exist for patients with heavily proteinuric CKD. Decisions made with regard to continuing or stopping ACE inhibitors or ARBs, or switching to different medication classes, therefore should not be taken lightly.

In addition to the potential risks to individual patients, inappropriate cessation of drugs could increase the need for hospitalization and place additional pressure on already-limited medical resources. Decisions made autonomously by patients to discontinue their medications based on weak evidence risks loss of trust in the medical profession, reduced drug adherence in general and poorer control of chronic disease. Considering the available evidence and the potential for unintended adverse consequences, numerous international and national organizations have now released statements supporting the continued use of ACE inhibitors and ARBs according to established guidelines in patients with mild COVID-19, unless otherwise medically indicated $^{10}$. These recommendations will be updated rapidly if and when appropriate evidence comes to light.

\section{Moving forward}

It is vitally important that clinicians, basic researchers and epidemiologists all work together to obtain, analyse and interpret data as they become available in order to fully assess the contribution, if any, of ACE inhibitors and ARBs to this pandemic. These efforts require a thorough understanding of the ACE2-Ang(1-7) and ACE-Ang II pathways in patients with SARS-CoV-2 and COVID-19. Such studies will provide greater insights into the impact of the virus on the RAS and will therefore have important implications for clinical management. We also need to be cautious not to draw inappropriate conclusions from observational studies and welcome the rapid initiation of clinical trials (for example, NCT04311177 and NCT04312009) that aim to provide conclusive evidence in this area.

1. Zhou, F. et al. Clinical course and risk factors for mortality of adult inpatients with COVID-19 in Wuhan, China: a retrospective cohort study. Lancet 395, 1054-1062 (2020).

2. Hoffmann, M. et al. SARS-CoV-2 cell entry depends on ACE2 and TMPRSS2 and is blocked by a clinically proven protease inhibitor. Cell https://doi.org/10.1016/j.cell.2020.02.052 (2020).

3. South, A. M. et al. Fetal programming and the angiotensin- $(1-7)$ axis: a review of the experimental and clinical data. Clin. Sci. 133 55-74 (2019).

4. Sparks, M. A. et al. Classical renin-angiotensin system in kidney physiology. Compr. Physiol. 4, 1201-1228 (2014).

5. Kuba, K. et al. A crucial role of angiotensin converting enzyme 2 (ACE2) in SARS coronavirus-induced lung injury. Nat. Med. 11, 875-879 (2005).

6. Ferrario, C. M. et al. Effect of angiotensin-converting enzyme inhibition and angiotensin II receptor blockers on cardiac angiotensin-converting enzyme 2. Circulation 111, 2605-2610 (2005).

7. Soler, M. J. et al. Localization of ACE2 in the renal vasculature: amplification by angiotensin II type 1 receptor blockade using telmisartan. Am. J. Physiol. Renal Physiol. 296, F398-F405 (2009).

8. Burrell, L. M. et al. Myocardial infarction increases ACE2 expression in rat and humans. Eur. Heart J. 26, 369-375 (2005).

9. Ramchand, J. et al. Elevated plasma angiotensin converting enzyme 2 activity is an independent predictor of major adverse cardiac events in patients with obstructive coronary artery disease. PLoS One 13, e0198144 (2018).

10. Sparks, M. A. et al. The coronavirus conundrum: ACE2 and hypertension edition. NephJC http://www.nephjc.com/news/ covidace2 (2020).

\section{Acknowledgements}

The authors thank the members of the COVID-19 and ACE2 in Cardiovascular, Lung, and Kidney Working Group for critical discussion of the relevant literature: D. Batlle, V. Bhalla, L. M. Burrell, J. B. Byrd, J. Cohen, M. Luther, M. Rheault, M. J. Soler, P. Welling and S. Swaminathan.

\section{Competing interests}

The authors declare no competing interests. 